\title{
Telomere Length in Newborns is Related to Maternal Stress During Pregnancy
}

\author{
Tabea Sarah Send', Maria Gilles', Veryan Codd ${ }^{2}$, Isabell Wolf', Svenja Bardtke', Fabian Streit ${ }^{3}$, \\ Jana Strohmaier ${ }^{3}$, Josef Frank $^{3}$, Darja Schendel ${ }^{3}$, Mark W Sütterlin ${ }^{4}$, Matthew Denniff ${ }^{2}$, Manfred Laucht ${ }^{5,6}$, \\ Nilesh J Samani ${ }^{2}$, Michael Deuschle ${ }^{1}$, Marcella Rietschel ${ }^{3}$ and Stephanie H Witt*,3 \\ 'Department of Psychiatry and Psychotherapy, Central Institute of Mental Health, Medical Faculty Mannheim, University of Heidelberg, \\ Mannheim, Germany; ${ }^{2}$ Department of Cardiovascular Sciences, University of Leicester, Leicester, UK; ${ }^{3}$ Department of Genetic Epidemiology in \\ Psychiatry, Central Institute of Mental Health, Medical Faculty Mannheim, University of Heidelberg, Mannheim, Germany; ${ }^{4}$ Department of \\ Gynecology and Obstetrics, University Medical Center Mannheim, University of Heidelberg, Mannheim, Germany; ${ }^{5}$ Department of Child and \\ Adolescent Psychiatry and Psychotherapy, Central Institute of Mental Health, Medical Faculty Mannheim, University of Heidelberg, Mannheim, \\ Germany; ${ }^{6}$ Department of Psychology, University of Potsdam, Potsdam, Germany
}

\begin{abstract}
Telomere length (TL) is a marker of biological aging, and numerous studies have shown associations between TL and somatic or psychiatric disorders. Research also indicates an association between maternal stress during pregnancy and TL in the offspring. The present study investigated possible associations between TL and: (I) maternal perceived stress during pregnancy; (2) a maternal lifetime history of psychiatric disorder (lifetime PD); and (3) paternal age. TL was analyzed in 319 newborns and 318 mothers from a predominantly Caucasian sample ( $n=273$ Caucasian newborns and $n=274$ Caucasian mothers). Two key findings were observed. First, maternal perceived stress during pregnancy was associated with shorter telomeres in newborns but not with maternal TL. Second, maternal lifetime PD was associated with shorter maternal telomeres, but not with TL in newborns. Paternal age was not associated with TL in newborns. The finding that maternal stress during pregnancy is associated with shorter telomeres in newborns supports the results of smaller previous studies. The fact that a relation between maternal prenatal stress and TL was observed in the offspring but not in mothers may be attributable to a high vulnerability to stress during intrauterine development of a maturing organism. To our knowledge, this is the largest study to date to show that maternal stress during pregnancy but not maternal lifetime PD is associated with shorter telomeres in the offspring. Neuropsychopharmacology (2017) 42, 2407-24I3; doi:10.1038/npp.2017.73; published online 10 May 2017
\end{abstract}

\section{INTRODUCTION}

Telomeres are essential to the maintenance of chromosomal integrity, and consist of repeated DNA-sequences that cap and protect eukaryotic chromosomes. Telomeres shorten with each cell division, and eventually reach a critical length. This in turn leads to cellular senescence or apoptosis. Telomeres shorten substantially with increasing age, and telomere length (TL) is thus a bioindicator of aging. Numerous studies have shown an association between TL and higher mortality and morbidity such as in patients with somatic and psychiatric disorders (Bojesen, 2013; Kimura et al, 2008; Lindqvist et al, 2015). The latter include major depression and post-traumatic stress disorder (Darrow et al, 2016).

TL displays wide interindividual variability, and this is attributable to both, heritability and environment. Heritability

\footnotetext{
*Correspondence: Dr SH Witt, Department of Genetic Epidemiology in Psychiatry, Central Institute of Mental Health, Medical Faculty Mannheim, J5, 68159 Mannheim, Germany, Tel.: +49 62I 1703 6056, Fax: +4962117036055

E-mail: stephanie.witt@zi-mannheim.de

Received 18 October 2016; revised 24 February 2017; accepted 6 April 2017; accepted article preview online II April 2017
}

accounts for up to $70 \%$ of the variance in TL (Broer et al, 2013; Hjelmborg et al, 2015). However, genetic variants identified to date in candidate gene and genome-wide association studies explain only a small proportion of the observed variance in TL. Women have longer telomeres than men, and research suggests that this difference may be present at birth (FactorLitvak et al, 2016). Several studies have demonstrated that paternal age is associated with TL in the offspring, with children of older fathers having longer telomeres (Broer et al, 2013). Individual variation of TL is a function of TL at birth and the subsequent attrition rate. In this context, research suggests that TL in early life is a more important predictor of TL in later life than attrition across the life span (Factor-Litvak et al, 2016; Heidinger et al, 2012). Factor-Litvak et al (2016) conclude that the effects of environmental factors during adulthood are 'small compared with the effect of the variation of leucocyte TL across newborns'.

Besides genetic factors, TL is also influenced by environmental conditions. For example, telomeres are highly sensitive to damage through alkylation, ultraviolet irradiation, and oxidative stress (von Zglinicki, 2002). Research has shown that stressful life events may lead to increased oxidative stress (Epel et al, 2004; Schiavone et al, 2015), 
and they may impact TL via this mechanism. This effect is most pronounced when stressful life events occur in childhood, which points to childhood as sensitive period (Oliveira et al, 2016; Osler et al, 2016). However, the literature is not entirely consistent (Glass et al, 2010; Verhoeven et al, 2015). Recent studies indicate that already maternal stress during pregnancy is associated with reduced TL in the offspring: Entringer et al (2013) examined mother-child dyads $(n=27)$, and found that psychosocial stress during pregnancy was associated with reduced TL in the newborns' cord blood. Two other studies focused on critical life events such as loss of a family member experienced by the mother during the prenatal period: A prospective cohort study with 24 motherchild dyads found an association between critical life events during pregnancy and shorter telomeres in the newborns' cord blood (Marchetto et al, 2016), and a retrospective design in $n=94$ subjects found an association between critical life events and lower TL in young adults (Entringer et al, 2011). The latter showed a more pronounced effect in females (Entringer $e t a l$, 2011). Shalev et al (2014) investigated a cohort of 38-year-old individuals and found that reduced TL was associated with a history of perinatal complications, such as maternal diabetes, low birth weight, and low Apgar scores, implying possible relevance of these early conditions in later life.

On the basis of these findings, Entringer et al (2015) concluded that telomere biology is an important mechanism in terms of fetal programming, and that the intrauterine phase of life may be particularly sensitive to environmental effects.

The aim of the present study was to investigate possible effects on TL in newborns/mothers of: (1) maternal perceived stress during pregnancy; and (2) a maternal lifetime history of psychiatric disorder (lifetime PD). The analyses were performed in a large, well-characterized and prospectively recruited cohort of newborns. Additional analyses were conducted to determine possible associations of newborns' TL with (3) paternal age.

\section{MATERIALS AND METHODS}

\section{Study Design and Sample}

The total sample comprised 410 mother-newborn dyads. TL was measured in 319 newborns and 318 mothers. Of these, 273 newborns and 274 mothers were Caucasian. The mothers were recruited during the third trimester of pregnancy (4-8 weeks prior to term) during attendance at a routine pre-delivery hospital registration appointment. Recruitment was performed between October 2010 and March 2013 at three obstetric clinics in the Rhine-Neckar Region of Germany in Mannheim and Ludwigshafen. The women were informed about the study (Pre-, Peri-, and Postnatal Stress: Epigenetic Impact on DepressiON; POSEIDON, see also Dukal et al, 2015; Nieratschker et al, 2014) by a study researcher, and provided with a printed flyer. The latter outlined the study design, which encompassed three measurement time-points: the end of the third trimester of pregnancy, immediately post delivery, and 6 months post delivery. The POSEIDON protocol included interviews, questionnaires, and the collection of biomaterial. An overview of the measures and biological samples collected at each time point is provided in Table 1 .

The following maternal inclusion criteria were applied: age 16-45 years; main caregiver; and German-speaking. Exclusion criteria for mothers were: positive hepatitis $\mathrm{B}$, hepatitis C, or human immunodeficiency virus (HIV) status; any current psychiatric disorder requiring inpatient treatment; any history or current diagnosis of schizophrenia/psychotic disorder; any substance dependency other than nicotine during pregnancy.

Exclusion criteria for newborns were: gestational age $<32$ weeks; birth weight $<1500 \mathrm{~g}$; multiple birth; and the presence of any congenital disease, malformation, deformation, and/or chromosomal abnormality.

Table I Phenotypic Assessment of Mothers and Infants Early in Life

\begin{tabular}{|c|c|c|}
\hline & Prenatal / third trimenon & Perinatal / birth \\
\hline Exposure to early life stress & $\begin{array}{l}\text { Perceived stress (PSS) } \\
\text { Prenatal distress (PDQ) } \\
\text { Life events (LES) } \\
\text { Social support (Soz-U) } \\
\text { Socio-demographic data } \\
\text { Maternal health risk behavior (eg smoking) } \\
\text { Psychosocial risks }\end{array}$ & $\begin{array}{l}\text { Pre- and perinatal complications } \\
\text { Perinatal stressors (eg asphyxia, cesarean, preterm birth) } \\
\text { Pregnancy and obstetric history (birth weight, gestational age, birth } \\
\text { complications) }\end{array}$ \\
\hline $\begin{array}{l}\text { Maternal mental \& physical } \\
\text { health }\end{array}$ & $\begin{array}{l}\text { Maternity log data } \\
\text { Semi-standardized neuropsychiatric diagnostic interview } \\
\text { (M.I.N.I.) } \\
\text { Depression screening (EPDS) } \\
\text { Anxiety screening (STAI-S, STAI-T, ASQ) } \\
\text { Anthropometry } \\
\text { Individual \& family history of metabolic and other medical } \\
\text { disorders }\end{array}$ & \\
\hline Biological samples & $\begin{array}{l}\text { Diurnal saliva cortisol (mother) } \\
\text { Saliva sample for genetic and epigenetic analysis (mother / } \\
\text { father) }\end{array}$ & $\begin{array}{l}\text { Cord blood for genetic and epigenetic analysis } \\
\text { Saliva sample for genetic and epigenetic analysis (infant) } \\
\text { Maternal and fetal placenta }\end{array}$ \\
\hline
\end{tabular}

Abbreviations: ASQ, anxiety screening questionnaire; EPDS, Edinburgh postnatal depression scale; LES, life experiences survey; M.I.N.I., Mini International

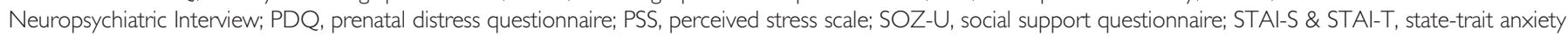
inventory. 
On the basis of the average number of deliveries per year for each hospital, an estimated 33\% of all eligible mothers agreed to participate in the study. Each female participant was reimbursed with 120 Euro. The study protocol was approved by the Ethics Committee of the Medical Faculty Mannheim of the University of Heidelberg, and the study was conducted in accordance with the Declaration of Helsinki. All women provided written informed consent prior to inclusion. Further sample characteristics are provided in Table 2 .

\section{Measures}

During the third trimester of pregnancy, structured interviews were conducted by study researchers to assess a broad range of environmental and sociodemographic risk factors, prenatal medical risk factors, general medical characteristics, and psychosocial risk factors (see Table 1). In addition, maternal saliva was collected.

The women were screened for current and lifetime psychopathology using the structured Mini International Neuropsychiatric Interview (M.I.N.I.; German version 5.0.0; Sheehan et al, 1998) and self-report.

Acute maternal stress was assessed with the Perceived Stress Scale (PSS; Cohen et al, 1983). This 14-item self-report questionnaire measures the individual's subjective evaluation of the stressfulness of situations encountered in the preceding month. Higher scores indicate higher perceived stress (see Table 3).

\section{Measurement of Telomere Length}

Umbilical cord blood was collected immediately after delivery by the attending midwife. The samples were drawn into ethylenediaminetetraacetic acid (EDTA)-coated tubes. For $n=311$ newborns, automated genomic DNA extraction was performed using the chemagic Magnetic Separation Module I (Chemagen Biopolymer-Technologie AG; Baesweiler; Germany). For $n=14$ further newborns, only a low volume of umbilical cord blood $(<2 \mathrm{ml})$ was obtained. For these samples, DNA was isolated using the QIAamp DNA Blood Midi Kit (Qiagen $\mathrm{GmbH}$, Hilden, Germany). All genomic DNA samples were stored at $-20^{\circ} \mathrm{C}$ prior to analysis. Maternal saliva was collected in Oragene DNA collection kits (DNA Genotek Inc., Ottawa, Ontario, Canada). Maternal DNA was extracted from saliva using the Oragene DNA purifier OG-L2P, in accordance with the Oragene DNA protocol (DNA Genotek Inc.). DNA isolation using the Chemagen module resulted in longer newborns' TL than DNA isolated using the QIAamp DNA Blood Midi Kit (Qiagen $\mathrm{GmbH})(p=0.029)$. Hence, we controlled for DNA extraction method in the analyses.

Relative TL was measured using an established quantitative PCR assay (Cawthon, 2002; Codd et al, 2010; Codd et al, 2013). This expresses TL as a ratio (T/S) of telomere repeat length ( $\mathrm{T}$ ) to a single-copy gene (S). Each sample was measured in duplicate for both $\mathrm{T}$ and $\mathrm{S}$. For both reactions, the amount was quantified relative to a calibrator sample included on every run (genomic DNA from the K562 cell line). For quality control samples were excluded and re-run if duplicate amplicons differed by $>0.2$ cycles or amplified outside linear range of the assay (Codd et al, 2010).
Table 2 Demographic Characteristics of Mothers and Infants Included in the Telomere Analysis (All Data: Mean \pm SD or Percentage)

\begin{tabular}{lc}
\hline Variable & Mean \pm SD or percentage \\
\hline Female infants (\%), with respect to $n=319$ & $52 \%$ \\
newborns & $31.5 \pm 5.0$ \\
Maternal Age (in years) & $34.7 \pm 6.3$ \\
Paternal Age (in years) & \\
\hline
\end{tabular}

Table 3 Maternal Psychopathology and Perceived Stress (Self-Report; All Data: Mean \pm SD or Percentage)

\begin{tabular}{|c|c|}
\hline Variable & Percentage or mean \pm SD \\
\hline \multicolumn{2}{|l|}{ Maternal psychopathology ${ }^{\mathrm{a}}$} \\
\hline Lifetime psychiatric disorder (\%) & $44 \%$ \\
\hline None & $56 \%$ \\
\hline Depressive disorder & $10.5 \%$ \\
\hline Adjustment disorder & $8.6 \%$ \\
\hline Anxiety disorder & $5.9 \%$ \\
\hline Eating disorder & $3.1 \%$ \\
\hline Substance abuse and misuse & $1.5 \%$ \\
\hline Other psychiatric disorder & $1.5 \%$ \\
\hline At least two different disorders specified ${ }^{b}$ & $13.9 \%$ \\
\hline \multicolumn{2}{|l|}{ Maternal perceived stress } \\
\hline Score on the Perceived Stress Scale (PSS) & $21.5 \pm 8.4$ \\
\hline
\end{tabular}

To ensure the absence of assay drift and to assess reproducibility of the measurement, 128 samples were measured on two separate occasions. The mean coefficient of variation between repeated T/S measurements was $2.72 \%$. TL was quantified in 15 different batches. There was a small but significant effect of the batch on TL $(p=0.036$ for newborns, $r^{2}=0.08 ; p=0.016$ for mothers, $\left.r^{2}=0.09\right)$. Hence, we controlled for batch effects in the analyses. TL measurements were obtained for 319 newborns (164 females, 155 males) and 318 mothers. Overall, 313 complete mother-child-dyads were included in the analyses. At the time of writing, no analysis of paternal TL has been performed.

\section{Statistical Analysis}

TL in newborns. Regression models were used to analyze the association between newborn TL and (1) maternal prenatal perceived stress, (2) maternal lifetime PD, and (3) paternal age. Newborn TL was residualized to account for batch effects and DNA extraction method. The analysis regarding paternal age was conducted with and without additional correction for maternal age. The analyses (1) and (2) were then repeated using residualized maternal TL as a further predictor. Due to lack of power, no interaction effects were added to the regression models. 
TL in mothers. The analyses were conducted likewise to those in newborns to analyze the association between maternal TL and (1) maternal prenatal stress, and (2) maternal lifetime PD. TL in mothers was residualized to account for batch effects. The analyses were repeated using maternal age as a predictor.

All statistical analyses were carried out with IBM SPSS Statistics (Version 22).

\section{RESULTS}

Telomeres were significantly longer in girls than in boys $\left(r^{2}=0.03, p=0.005\right)$, supporting previous evidence that TL differs between genders at birth (see introduction). As expected, a positive association was found between maternal $\mathrm{TL}$ and TL in the offspring $(\beta=0.31, p<0.001)$. The relationship between maternal TL and TL in the offspring was similar for both sexes (for females: $\beta=0.34, p<0.001$; for males $\beta=0.27, p=0.001)$. Maternal age showed a trend towards a negative relation with maternal TL $(\beta=-0.11$; $p=0.062$ ). Details of these analyses are provided in Tables 4 and 5. The reported standardized regression weights $\beta$ correspond to $r^{2}=0.10$ for the association between maternal $\mathrm{TL}$ and newborn TL, and to $r^{2}=0.01$ for the association between maternal age and maternal TL.

\section{Maternal Perceived Stress During Pregnancy and TL}

Maternal perceived stress during pregnancy was associated with TL in newborns $(\beta=-0.14, p=0.015$, see Table 5$)$. Inclusion of maternal TL as a predictor in the analyses of newborn TL did not impact on the results (see Supplementary Table 1). Maternal perceived stress during pregnancy was not associated with maternal TL. Inclusion of maternal age as a predictor in the analyses of maternal TL did not impact on the results (see Supplementary Table 2). The reported standardized regression weight $\beta$ corresponds to $r^{2}=0.02$ for the association between perceived stress and newborn TL.

\section{Maternal Lifetime PD and TL}

Maternal lifetime PD (self-report) was not associated with TL in newborns, but only shortly failed to show a significant association with shorter maternal telomeres $(\beta=-0.11$, $p=0.055$, see Table 5 as well as Supplementary Table 1 ).

Table 4 Telomere Length (TL) in Newborns and Mothers

\begin{tabular}{|c|c|c|}
\hline Telomere length in & Mean \pm SD $^{\mathrm{a}}$ & $p$ value $^{b}$ \\
\hline Newborns $(T / S)^{c}$ & $4.1 \pm 0.6$ & $N / A$ \\
\hline Female newborns & $4.2 \pm 0.6$ & 口 05 米米 \\
\hline Male newborns & $4.0 \pm 0.6$ & 0.005 \\
\hline Mothers $(T / S)^{c}$ & $3.0 \pm 0.5$ & $N / A$ \\
\hline \multicolumn{3}{|c|}{$\begin{array}{l}\text { a Mean TL and standard deviation. } \\
{ }^{\mathrm{b}} \mathrm{P} \text {-value indicating the significance of the difference in mean } \mathrm{TL} \text { of female and } \\
\text { male subgroup; significant } p \text {-values are indicated by } * \text { * for } p<=0.0 \mathrm{l} \text {. } \\
{ }^{\mathrm{C}} \mathrm{T} / \mathrm{S} \text { expresses telomere length as the ratio of telomere repeat length }(\mathrm{T}) \text { to a } \\
\text { single copy gene (S); Analyses were carried out with newborn } T L \text { values } \\
\text { residualized for batch effects and DNA extraction method and with maternal TL } \\
\text { values residualized for batch effects. }\end{array}$} \\
\hline
\end{tabular}

Neuropsychopharmacology
Inclusion of maternal age as a predictor in the analyses of maternal TL changed the results slightly $(\beta=-0.11$, $p=0.050$, see Supplementary Table 2). A highly significant correlation was found between maternal lifetime PD (assessed using the M.I.N.I.) and maternal lifetime PD (assessed by self-report): $r=0.53, p<0.001$. However, no association was found between diagnosis according to the M.I.N.I. and either maternal TL or newborn TL.

\section{Paternal Age and TL}

Paternal age was not associated with newborn TL $(\beta=0.06$, $p=0.328)$. Moreover, no association could be observed when the model was additionally corrected for maternal age (see Supplementary Table 1).

\section{DISCUSSION}

The present study reports an association between maternal perceived psychosocial stress during pregnancy and shorter telomeres in the largest sample of newborns to date. It substantiates previous findings in smaller samples (Entringer et al, 2013; Marchetto et al, 2016). Our finding of an association between maternal lifetime PD (self-report) and shorter maternal telomeres is also in accordance with the literature (Lindqvist et al, 2015). This association was not found for the M.I.N.I. diagnoses. Although the M.I.N.I. is more objective than the self-report, it is also less sensitive, since it classifies a total of only 17 psychiatric disorders. Maternal lifetime PD did not impact on TL in newborns. Moreover, no association was found between maternal stress during pregnancy and maternal TL. This is consistent with a previous meta-analysis, which revealed only a very small association between perceived stress (measured over the preceding month) and adult TL (Mathur et al, 2016).

The finding that acute stress measured with the PSS impacts on TL in the offspring but not in mothers may be attributable to an increased fetal vulnerability to stress during pregnancy, and underlines the potential importance of intrauterine effects in terms of newborn TL.

The PSS can be considered a broad measure for acute stress. It quantifies the individual's subjective evaluation of the stressfulness of situations encountered in the preceding month, including eg disturbances by daily hassles. In a metaanalysis performed by Nast et al (2013), the PSS was considered the best currently available instrument to measure the degree in which situations are regarded as unpredictable, uncontrollable, and burdensome during the past month. In a second step, we tested two other measures of prenatal stress, the Prenatal Distress Questionnaire (PDQ, Yali and Lobel, 1999) and the Life Experiences Survey (LES, Sarason et al, 1978). These were highly correlated with the PSS. However, they were not significantly associated with newborn or maternal TL (see Supplementary Table 3).

Nicotine use during pregnancy showed no association with newborn TL. This implies that the association between acute stress and newborn TL was not mediated by smoking behavior during pregnancy.

Previous studies have reported that paternal factors such as paternal TL and age impact on TL in newborns. While no 
Table 5 Predictors of Telomere Length $(T L)$ in Newborns and Mothers

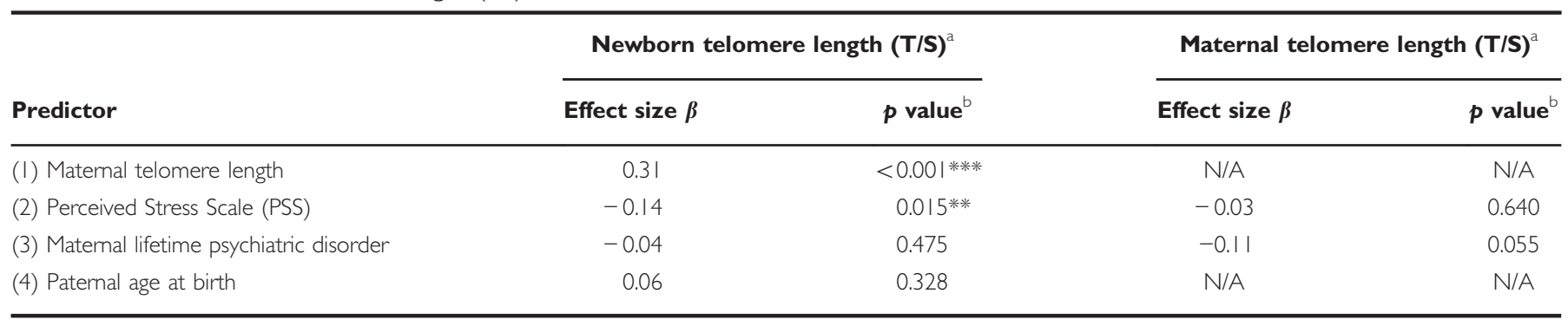

Regression analyses were carried out to assess: (I) association between maternal and newborn TL; (2) association between the Perceived Stress Scale (PSS) and

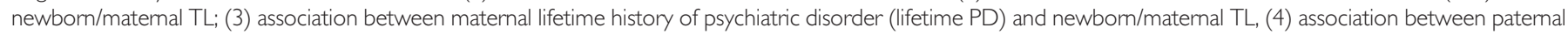
age and newborn TL.

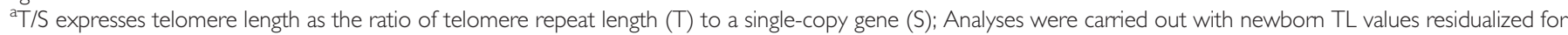
batch effects and DNA extraction method and with maternal TL values residualized for batch effects; no further predictors were included in the analyses.

bignificant $p$-values are indicated by *** for $p<0.01$; and **** for $p<0.001$.

data were available to date concerning paternal TL, we examined whether paternal age was associated with shorter telomeres of the newborns. No significant association was found between paternal age and newborns TL. However, the average effect size described in a recent meta-analysis is comparatively low (corresponding to $r=0.055$; Broer et al, 2013) and the power to detect such an effect in our sample was $16 \%$.

There are several limitations of our approach. The exclusion of women with a psychiatric disorder requiring in-patient treatment and substance dependency (except nicotine dependency) during pregnancy may have led to an underestimation of the association between maternal psychiatric history and maternal TL. It may as well have led to the overlooking of an association with newborn TL. Therefore, the present data do not exclude the possibility that severe maternal psychiatric disorder is associated with newborn TL. However, although mothers with a severe psychiatric disorder (who might be more distressed than women with no psychiatric disorder) were excluded, an association between perceived stress during pregnancy and newborn TL was demonstrated. Furthermore, our sample was recruited during the third trimester of pregnancy. We were therefore unable to investigate specific influences during earlier phases of fetal development. However, a recent study showed in a large sample that PSS scores are generally stable over time during pregnancy (Bann et al, 2017). The analysis of paternal age had the limitation that no data were available concerning paternal TL.

In conclusion, our study is the largest prospective cohort study to date to show that maternal perceived stress during pregnancy is associated with shorter telomeres in the offspring and that maternal lifetime PD seems to have no impact on telomeres in the offspring. It has been shown that environmental factors during specific time frames may affect the offspring but not the mothers (Lupien et al, 2009). Also, there are vulnerable phases in which environmental effects are more detrimental than in other phases. For example, this has been prominently shown in the Dutch Hunger Winter Study for prenatal (Beijers et al, 2014; Susser and Lin, 1992) as well as for adolescent phases (Fransen et al, 2016). As Barker and many others have reported these influences contribute to later disease such as coronary heart disease and schizophrenia (Barker, 1995, 2002; Barker and Thornburg, 2013; Plana-Ripoll et al, 2016; Susser and Lin, 1992). Reduced leucocyte TL was related to cardiovascular disease (Haycock et al, 2014; Jansen et al, 2014). Thus, the effect of prenatal stress on the risk of heart disease could be mediated by prenatal stress-associated low TL.

Although the meaning of the reported differences in TL for later health is so far unclear, our findings underline the necessity to especially support women with increased risk to experience stress during pregnancy.

TL at birth is one of the parameters that define the oldest potential age of an individual, as based on his/her genetic endowment under environmental circumstances that reduce extrinsic mortality (Kimura et al, 2008). Therefore, determining factors with potential effects on TL in newborns is an important research endeavor. Although there is evidence that TL plays a causal role in some age-related diseases, including coronary artery disease and some cancers (Codd et al, 2013; Walsh et al, 2015), the issue of whether TL plays a wider causal or adaptive role in mortality, is unclear. However, with respect to our study, even if TL represents only a biomarker for prenatal stress, its value in identifying 'preaged' children could be of relevance in terms of early intervention.

\section{FUNDING AND DISCLOSURE}

The study was supported by a Era-Net Neuron grant to MD, MR, and ML, the German Federal Ministry of Education and Research (BMBF) through the Integrated Network IntegraMent (Integrated Understanding of Causes and Mechanisms in Mental Disorders), under the auspices of the e:Med Programme (grant 01ZX1314G to MR) and by a grant of the Dietmar-Hopp Foundation. The study was supported by the German Research Foundation (DFG; grant FOR2107; RI908/11-1 to MR; WI3429/3-1 to SHW). VC and NJS are supported by the British Heart Foundation and NJS is a UK NIHR Senior Investigator. MD served as PI in phase II and III studies of Johnson \& Johnson, Lilly and Roche. He participated in advisory boards of Bristol-Myers Squibb, Lundbeck and Otsuka and received speaker fees from Bristol-Myers Squibb, Janssen, Lundbeck, Mundipharma and Otsuka. The remaining authors declare no conflict of interest. 


\section{ACKNOWLEDGMENTS}

We are very grateful for the essential contributions of the midwives, and would like to thank all families for their participation.

\section{REFERENCES}

Bann CM, Parker CB, Grobman WA, Willinger M, Simhan HN, Wing DA et al (2017). Psychometric properties of stress and anxiety measures among nulliparous women. J Psychosom Obstet Gynaecol 38: 53-62.

Barker DJ (1995). Fetal origins of coronary heart disease. Bmj 311: 171-174.

Barker DJ (2002). Fetal programming of coronary heart disease. Trends Endocrinol Metab 13: 364-368.

Barker DJ, Thornburg KL (2013). The obstetric origins of health for a lifetime. Clin Obstet Gynecol 56: 511-519.

Beijers R, Buitelaar JK, de Weerth C (2014). Mechanisms underlying the effects of prenatal psychosocial stress on child outcomes: beyond the HPA axis. Eur Child Adolesc Psychiatry 23: 943-956.

Bojesen SE (2013). Telomeres and human health. J Intern Med 274: 399-413.

Broer L, Codd V, Nyholt DR, Deelen J, Mangino M, Willemsen G et al (2013). Meta-analysis of telomere length in 19,713 subjects reveals high heritability, stronger maternal inheritance and a paternal age effect. Eur J Hum Genet 21: 1163-1168.

Cawthon RM (2002). Telomere measurement by quantitative PCR. Nucleic Acids Res 30: e47.

Codd V, Mangino M, van der Harst P, Braund PS, Kaiser M, Beveridge AJ et al (2010). Common variants near TERC are associated with mean telomere length. Nat Genet 42: 197-199.

Codd V, Nelson CP, Albrecht E, Mangino M, Deelen J, Buxton JL et al (2013). Identification of seven loci affecting mean telomere length and their association with disease. Nat Genet 45: 422-427, 427e421-422.

Cohen S, Kamarck T, Mermelstein R (1983). A global measure of perceived stress. J Health Soc Behav 24: 385-396.

Darrow SM, Verhoeven JE, Revesz D, Lindquist D, Penninx BW, Delucchi KL et al (2016). The association between psychiatric disorders and telomere length: a meta-analysis involving 14,827 persons. Psychosom Med 78: 776-787.

Dukal H, Frank J, Lang M, Treutlein J, Gilles M, Wolf IA et al (2015). New-born females show higher stress- and genotypeindependent methylation of SLC6A4 than males. Borderline Personal Disord Emot Dysregul 2: 8.

Entringer S, Buss C, Wadhwa PD (2015). Prenatal stress, development, health and disease risk: A psychobiological perspective-2015 Curt Richter Award Paper. Psychoneuroendocrinology 62: 366-375.

Entringer S, Epel ES, Kumsta R, Lin J, Hellhammer DH, Blackburn $\mathrm{EH}$ et al (2011). Stress exposure in intrauterine life is associated with shorter telomere length in young adulthood. Proc Natl Acad Sci USA 108: E513-E518.

Entringer S, Epel ES, Lin J, Buss C, Shahbaba B, Blackburn EH et al (2013). Maternal psychosocial stress during pregnancy is associated with newborn leukocyte telomere length. Am J Obstet Gynecol 208: 134.e131-137.

Epel ES, Blackburn EH, Lin J, Dhabhar FS, Adler NE, Morrow JD et al (2004). Accelerated telomere shortening in response to life stress. Proc Natl Acad Sci USA 101: 17312-17315.

Factor-Litvak P, Susser E, Kezios K, McKeague I, Kark JD, Hoffman $M$ et al (2016). Leukocyte telomere length in newborns: implications for the role of telomeres in human disease. Pediatrics 137: e20153927.

Fransen HP, Peeters PH, Beulens JW, Boer JM, de Wit GA, Onland-Moret NC et al (2016). Exposure to famine at a young age and unhealthy lifestyle behavior later in life. PloS ONE 11: e0156609.

Glass D, Parts L, Knowles D, Aviv A, Spector TD (2010). No correlation between childhood maltreatment and telomere length. Biol Psychiatry 68: e21-e22.

Haycock PC, Heydon EE, Kaptoge S, Butterworth AS, Thompson A, Willeit P (2014). Leucocyte telomere length and risk of cardiovascular disease: systematic review and meta-analysis. BMJ 349: g4227.

Heidinger BJ, Blount JD, Boner W, Griffiths K, Metcalfe NB, Monaghan P (2012). Telomere length in early life predicts lifespan. Proc Natl Acad Sci USA 109: 1743-1748.

Hjelmborg JB, Dalgard C, Moller S, Steenstrup T, Kimura M, Christensen $\mathrm{K}$ et al (2015). The heritability of leucocyte telomere length dynamics. J Med Genet 52: 297-302.

Jansen H, Samani NJ, Schunkert H (2014). Mendelian randomization studies in coronary artery disease. Eur Heart J 35: 1917-1924.

Kimura M, Hjelmborg JV, Gardner JP, Bathum L, Brimacombe M, $\mathrm{Lu} \mathrm{X}$ et al (2008). Telomere length and mortality: a study of leukocytes in elderly Danish twins. Am J Epidemiol 167: 799-806.

Lindqvist D, Epel ES, Mellon SH, Penninx BW, Revesz D, Verhoeven JE et al (2015). Psychiatric disorders and leukocyte telomere length: underlying mechanisms linking mental illness with cellular aging. Neurosci Biobehav Rev 55: 333-364.

Lupien SJ, McEwen BS, Gunnar MR, Heim C (2009). Effects of stress throughout the lifespan on the brain, behaviour and cognition. Nat Rev Neurosci 10: 434-445.

Marchetto NM, Glynn RA, Ferry ML, Ostojic M, Wolff SM, Yao R et al (2016). Prenatal stress and newborn telomere length. Am J Obstet Gynecol 215: 94 e91-94 e98.

Mathur MB, Epel E, Kind S, Desai M, Parks CG, Sandler DP et al (2016). Perceived stress and telomere length: a systematic review, meta-analysis, and methodologic considerations for advancing the field. Brain Behav Immun 54: 158-169.

Nast I, Bolten M, Meinlschmidt G, Hellhammer DH (2013). How to measure prenatal stress? A systematic review of psychometric instruments to assess psychosocial stress during pregnancy. Paediatr Perinat Epidemiol 27: 313-322.

Nieratschker V, Massart R, Gilles M, Luoni A, Suderman MJ, Krumm B et al (2014). MORC1 exhibits cross-species differential methylation in association with early life stress as well as genome-wide association with MDD. Transl Psychiatry 4: e429.

Oliveira BS, Zunzunegui MV, Quinlan J, Fahmi H, Tu MT, Guerra RO (2016). Systematic review of the association between chronic social stress and telomere length: A life course perspective. Ageing Res Rev 26: 37-52.

Osler M, Bendix L, Rask L, Rod NH (2016). Stressful life events and leucocyte telomere length: Do lifestyle factors, somatic and mental health, or low grade inflammation mediate this relationship? Results from a cohort of Danish men born in 1953. Brain Behav Immun 58: 248-253.

Plana-Ripoll O, Liu X, Momen NC, Parner E, Olsen J, Li J (2016). Prenatal exposure to maternal stress following bereavement and cardiovascular disease: a nationwide population-based and sibling-matched cohort study. Eur J Prev Cardiol 23: 1018-1028.

Sarason IG, Johnson JH, Siegel JM (1978). Assessing the impact of life changes: development of the Life Experiences Survey. J Consult Clin Psychol 46: 932-946.

Schiavone S, Colaianna M, Curtis L (2015). Impact of early life stress on the pathogenesis of mental disorders: relation to brain oxidative stress. Curr Pharm Des 21: 1404-1412.

Shalev I, Caspi A, Ambler A, Belsky DW, Chapple S, Cohen HJ et al (2014). Perinatal complications and aging indicators by midlife. Pediatrics 134: e1315-e1323.

Sheehan DV, Lecrubier Y, Sheehan KH, Amorim P, Janavs J, Weiller E et al (1998). The Mini-International Neuro- 
psychiatric Interview (M.I.N.I.): the development and validation of a structured diagnostic psychiatric interview for DSM-IV and ICD-10. J Clin Psychiatry 59(Suppl 20): 22-33.

Susser ES, Lin SP (1992). Schizophrenia after prenatal exposure to the Dutch Hunger Winter of 1944-1945. Arch General Psychiatry 49: 983-988.

Verhoeven JE, van Oppen P, Puterman E, Elzinga B, Penninx BW (2015). The association of early and recent psychosocial life stress with leukocyte telomere length. Psychosom Med 77: 882-891. von Zglinicki T (2002). Oxidative stress shortens telomeres. Trends Biochem Sciences 27: 339-344.

Walsh KM, Codd V, Rice T, Nelson CP, Smirnov IV, McCoy LS et al (2015). Longer genotypically-estimated leukocyte telomere length is associated with increased adult glioma risk. Oncotarget 6: 42468-42477.

Yali AM, Lobel M (1999). Coping and distress in pregnancy: an investigation of medically high risk women. J Psychosom Obstet Gynaecol 20: 39-52.

Supplementary Information accompanies the paper on the Neuropsychopharmacology website (http://www.nature.com/npp) 